\title{
A learning development-faculty collaborative exploration of postgraduate research student mental health in a UK university
}

\author{
Russell Delderfield \\ University of Bradford
}

\section{Kirsten Riches-Suman}

University of Bradford

\section{Mathias Ndoma-Egba}

University of Bradford

\section{James Boyne}

University of Huddersfield

\section{Abstract}

Mental ill-health is an escalating problem in higher education. Not only does this impact students' ability to learn, it can lead to poor completion, with learners opting to withdraw from studies, even if attainment has been satisfactory. The aim of this study was to gain insight about perceptions of poor mental health from postgraduate research students in a diverse UK university and canvas opinion regarding how the University could improve this. A short, pragmatic survey with basic quantitative and qualitative responses was distributed. This was analysed by a team comprising the learning developer responsible for postgraduate researcher learning development, academics and a doctoral student. The study found that poor mental health was evident, with over three quarters of respondents reporting some experience of mental ill-health. We identified five areas in need of attention: University Systems, Supervisor Training, Well-being Monitoring, Building Networks, and Finance. Sources of University-based stress were finance, administrative support, and an environment where a perception that poor mental health was an expectation rather than a problem was experienced. Students preferred to access support outside the academic environment. This is the first study of its kind at a diverse, plateglass UK university, to consider research student mental ill-health, with a staff-student team working with data, and the learning developer spear-heading changes across 
postgraduate research. These findings have already influenced university strategy, staff training, and induction practices. The synthesis of the five areas could be used to visualise where further work is needed to improve mental health in these learners.

Keywords: postgraduate research students; PGR; doctoral learning; mental health; learning development and supervision; researcher development framework.

\section{Introduction}

The mental health of students in higher education (HE) has an increasingly elevated profile. What began as a tacit acknowledgement that students experience poor mental health has grown into an area of openly debated concern (Neves and Hillman, 2017). Academics with pastoral responsibilities are concerned at what they perceive to be a significant increase in the number of tutees experiencing mental health issues (Hughes et al., 2018). The most recent summary of student experiences of mental ill-health in 2017 (collating over 14,000 responses in the UK) found that positive mental health measures have showed a year-on-year decline since 2007, with students reporting lower levels of mental health than the national population using the UK's Office for National Statistics Data as comparator (Neves and Hillman, 2017). It is also known that those students with the greatest need (significant distress or particularly poor mental health) are the least likely to seek help or support (Gorczynski et al., 2017). Furthermore, a national initiative for student mental health support, the Step Change framework (Universities UK, 2017) reports an increase in suicide among students. These serious concerns are not unique to the UK, with studies across 21 countries analysing and evidencing mental health issues, suicidal thoughts and behaviours (Mascaskill, 2012; Evans et al., 2018; Mortier et al., 2018).

There is a starker message about mental health issues when specifically focussing on postgraduate researchers (PGR). Both Science and Nature have recently reflected on PGR mental health. The issues already outlined above have been deemed so severe that terms such as 'crisis' or the effects of 'indentured servitude' are employed (Pain, 2018; Editorial, 2018). The supervisory relationship is a key part of these experiences (Pole et al., 1997; Solem et al., 2011). In this context, supervision is configured as a complex learning and teaching relationship. Where supervision is deemed to be effective, students may flourish and overcome personal and professional adversity. Where supervision is 
poor, a student can feel the effects of a mental health condition more acutely or begin to develop a previously unexperienced condition (Pain, 2018; Editorial, 2018). Supervisors may struggle to appreciate the need for a clear work-life balance, as they too may be grappling with issues around stress and poor mental health caused by routine overworking (Kinman, 2008; Sang et al., 2015; Guthrie et al., 2017), perhaps a severe example of which would be the suicide of a Cardiff University academic, reported to be a 'wake-up call' to all in academia (Pells, 2018).

Students who undertake research degrees reportedly experience additional challenges that are not found when undertaking taught courses, including isolation, financial difficulties, impostor syndrome and an increasingly difficult employment landscape (Levecque et al., 2017). The mental health of these students may be indicative of the future state of a nation's ability to lead and deliver on much-needed research advancements. Recent work has highlighted that the persistent subjugation of the mental and physical well-being of students, in favour of cerebral attainment and output, has personal consequences. We are losing sight of a key element of doctoral learning: the 'production of new . . . selves' (Hopwood and Paulson, 2012, p.668), as we obsess over the production of a new thesis, instead.

A landmark UK report about PGR mental health was recently published (Metcalfe et al., 2018). Data reported suggest an escalation - in 2014 , only $0.9 \%$ of students declared a mental health issue in official returns. Yet, by 2017 the Postgraduate Research Experience Survey (PRES), a biennial survey conducted across PGR in HE, found that $3.3 \%$ of respondents declared a mental health condition. Metcalfe et al. (2018) make a compelling case for the incidence of mental health problems found in these learners but contrast this with the dearth of site-specific studies that investigate this phenomenon.

Doctoral learning is allegedly stressful to undertake, denoted by its 'trauma and neglect' (John and Denicolo, 2013, p.41), yet there can be benefits of this through the building and nurturing of personal resources and resilience, and the use of stress or anxiety as intrinsic motivators to effect progress and change. However, when mental health is not prioritised by departments and services, and 'working hard above all else' is the default learning culture (Metcalfe et al., 2018, p.22), this can lead to students feeling ashamed to admit difficulties to supervisory teams when problems arise. This is compounded by perceptions 
that Higher Education Institutions (HEIs) are reluctant to address complex or problematic supervisory relationships (Metcalfe et al., 2018).

Sources of strain for these learners include financial issues. Funding often covers project costs but grants (or 'stipends') can average out at lower than the minimum wage. Money for social activities that could have a restorative effect on mental health through self-care and connection is considered sparse (Metcalfe et al., 2018). There is also an expectation that undertaking a PhD means a poor work-life balance, and that working long hours is part of the experience. Students reported that supervisors appeared to think that the weekend was for further study. Some students felt they were being 'exploited' through overwork due to graduate teaching responsibilities and that they had little control over this. Finally, being confident about future career possibilities was linked with improved mental health (Cowling, 2017; Metcalfe et al., 2018).

It is important to be clear from the outset that there are two key concepts this paper relies upon. The first is that we all have mental health, and the quality of our mental health can 'ebb and flow', changing over time due to various factors (Mental Health Foundation, 2018). These factors may be socio-economic, cultural, environmental or physical in nature (World Health Organization, WHO, 2014). When referring to self-reported mental ill-health we are drawing on WHO's (2014) definitions, which suggest that the way people think, feel, and behave can be influenced for the worse by undiagnosed experiences of stress, anxiety or depression, as well as diagnosed conditions. There are some tensions in the use of terminology; for example, services and activities designed to improve mental health are often referred to as 'well-being' services in our institution and form part of a larger offer around physical health, as well as creating communities. We are mindful of Dhillon's (2018) assertions about the broad, unquestioning uptake of the concept of well-being and the dangers that this entails, namely that students should adapt to the status quo, but there can be little doubt the term currently pervades. We do not suggest that this term can be synonymously substituted for the concept of mental health, and efforts are made to apply consistency, but we recognise students' identification of differing terminology based on their experience of named services and resources in our $\mathrm{HEI}$, and this is reflected in our synthesis below. 
The second concept upon which our collaborative work in this study has relied is that of learning development (LD). This paper frames PGR as students (the terms learners and students are used interchangeably), with learning development being the overarching field that has much to offer in terms of how the personal development of our students is underpinned, implemented, and supported across an institution. LD itself encompasses the pedagogical, psycho-social and systemic aspects of higher education at all levels, with a clear ethos of 'creating a sense of belonging' for all (Stapleford, 2019, p.2). It is also the vocation of one of our authors, who has been an institutional member of the Association for Learning Development in Higher Education since membership was introduced (further detail about the LD role in our institution is offered below in the context section). All five of the LD values as available via the website and in Stapleford $(2019$, p.3) are in evidence here through:

- Our work with a PGR student in developing and writing up the study and our engagement with our PGR student body (Value 1).

- Our collaboration across roles, disciplines and institutional structures (Value 2).

- Sharing our practice, its insights and limitations for the benefit of others (Value 3).

- The experience leading to reflection and development between scientists (staff and student) and the learning developer, as we learn about our own students, our systems and practices, and even our writing and reporting conventions (Value 4).

- Our commitment to using scholarship to inform and question LD and its place in doctoral education (Value 5).

Our working together illuminated the advantages of such a collaboration. There is a 'twoway sharing of knowledge and expertise' between the learning developer and the faculty subject-specialists (Cairns et al., 2018, p.4), which is used to benefit the student experience - our shared goal. Thus, LD is suitable for our study's aims. This is important for what follows in the context of this study and the role of the learning developer at our university.

\section{Research aims}

A conclusion from a review of doctoral students' experience (John and Denicolo, 2013) was that there is a dearth of data from within specific institutions. Our aim was to discover 
whether students identified with mental health issues, and, more significantly for our immediate practices as learning developers and academics, where they turn for support. To our knowledge, this is the first preliminary survey study of its kind in a northern-UK institution. It represents an innovative, collaborative effort between the learning developer in charge of PGR development, faculty academics, and a student in making sense of PGR mental health. Whilst this collaboration is not suggested as an aim of our study, it was certainly a driver in our working together to produce this work. Finally, whilst it draws on a modest convenience sample, it provides site-specific data in a field that is deemed to be of high import but is aetiolated by lack of research-derived examples. This is an important contribution because recent reports regarding student mental health rarely contextualise these issues to an immediate HE environment, therefore, it is assumed rather than evidenced, that similar issues occur across institutions (see Ampaw and Jaeger, 2012, for work carried out on a single institution in the US). Such studies are rare in the UK. Our study's original contribution aims to address this sparsity of data by offering insights drawn from one local population of PGR students in a UK HEI.

\section{The context}

The University of Bradford is a UK plate-glass university situated in West Yorkshire. In the 2017-18 academic year, total enrolled numbers were 8,860 students, with $10 \%$ of our student body declaring a disability, including mental health conditions (under UK law these conditions form part of the protected characteristic of 'disability'). The PGR community is relatively small, with only 320 of these students studying a research degree leading to a doctoral award (such as a PhD or equivalent) at the time of our study. The institution has a diverse profile - whilst gender is relatively balanced, over $50 \%$ of our PGR are mature students and over $65 \%$ are international. Students are allocated a minimum of two research supervisors. The university makes a range of resources and services available to PGR students. There are library, careers, counselling, disability (including mental health) and language teams.

Faculty colleagues in this collaboration have reflected upon on how quickly subject specialists may become siloed within institutional structures and strictures that are perpetuated within their academic communities in terms of the accepted role of a research academic. The learning developer experiences fewer constraints. Indeed, given previous 
(and continuing?) debate about LD operating within a deficit model (Johnson, 2018), at Bradford, the opposite may be said to be the case. LD is agile, connected and 'liberated' from the typical structures used to organise academic teams and programmes. LD is at the forefront of PGR support, as a dedicated senior learning developer (one of the authors) not only offers one-to-one and group development, but also manages the Postgraduate Researcher Framework. This framework covers all aspects of doctoral student experience, bringing together faculties and support services in providing a holistic development and support programme. This programme of events ranges from formal research training to peer-support events, including our 'PGR Connect' project which is establishing systems for PGR mental health and well-being support. The framework encompasses the entire student lifecycle, including PGR induction through to doctorate completion, including scholarly writing skills and viva skills. He is involved in PRES outcomes action-planning across the institution and works actively with researchers and supervisors on issues arising around research culture, belonging, and research supervision. The learning developer delivers training and support to research supervisors as part of the university's commitment to improving PGR supervision.

In this way, the learning developer's role is simultaneously academic and non-academic, and moves between students, research supervisors, and professionals within support services. Whitchurch (2013) has described this lack of fixed positionality as a valuable benefit, a Third Space from which to work. The learning developer offers a 'service' that is founded upon the values above and is academically grounded. He holds a doctorate in his own field, thus having personal experience of the doctoral journey, and trains both staff and students in the craft of research and researcher identity. He also works at a senior level with key stakeholders, including support service managers, to inform and mould the future of the PGR provision at our HEI. Occupying a space that cannot be reduced to one of two binary positions (academic or non-academic), the learning developer can effect change for students across disciplines and practices, and can work from an authentic place, being able to help-without-constraint (Whitchurch, 2013) wherever he is able to do so, in a person-centred way (Delderfield and McHattie, 2018). Most UK HEls are reimagining their PGR experience, as the re-affirmation of the Researcher Development Concordat (Vitae, 2020a), requires research degrees to offer more than training in becoming a subject expert. In other words, the aforementioned obsession with thesisoutput is giving way to the development of a student's entire self as a high-level, reflective, 
adaptable, and adept problem-solver and thinker. The doctoral experience is becoming one of the changes to the selfhood of the researcher (Hopwood and Paulson, 2012), as much as developing insights into the researched. The learning developer is ideally situated to support individuals to develop, flourish and cope with the demands of doctoral life, as well as producing quality research. LD professionals help researchers to reflect and work on building skills to improve belonging and relationships with their learning communities (Parkes, 2018). As such, the findings presented below are being used to inform the continuous improvement of the PGR Framework and the learning developer's work with faculty research leads.

Pertinent to what follows below, we have recently been trialling a mentor system in one of our faculties, where learners are allocated an additional academic, unrelated to their project, with whom they can have regular contact to seek support for issues outside of their specific research, for example, concerns regarding supervision or personal strategies for coping with high-level study. This is currently a trial and may be implemented across the university by the learning developer working with our academics leading PGR in each faculty.

\section{Methods}

There has been work conducted to design an instrument that can evaluate the well-being of students from a mental health perspective (Juniper et al., 2012); given our topic, it is notable that no further newly-developed instruments have been published that aim to engage with PGR mental health. Considering this, and for our purposes, we needed something smaller in scale and easier to administer within a small community. A short, anonymous survey was composed and received ethical approval. It was distributed to all actively enrolled students on $5^{\text {th }}$ June and closed on $11^{\text {th }}$ June 2018, after which data was collated, analysed and discussed at the university's annual internal conference that same month.

We wish to make it clear that the survey was opportunistic and simplistic, in a straw pollstyle, using an opening in the student survey calendar. Questions were iteratively generated through collegiate discussions and framed by an assumption that poor mental health is prevalent in HEls. The survey comprised five questions (Figure 1). The free-form 
responses to the final question gave anonymous quotes; selected ones of which are presented in the results and discussion to illustrate proposed areas of improvement.

Responses to the questions were analysed by frequency count with some iterative qualitative analysis undertaken on the limited experiential comments offered by respondents. Said analysis consisted of an adaptation of Braun and Clarke's (2006) thematic analysis to explore trends and patterns in the personal comments and reflections. The resultant synthesis identified the main areas of import to our students (Figure 3) and the relationships between these. Researchers analysed in parallel, then explored consensus for shared interpretations. Fine-grained detail influencing our method's steps is available but is beyond the scope of this paper (Braun and Clarke, 2006; Maguire and Delahunt, 2017). 
Figure 1. Postgraduate researcher mental health. Structure of the short survey that was distributed to all PGR at the University of Bradford in June 2018.

Q1: During your postgraduate research, have you ever felt that you have developed any of the following?

Select as many as are applicable
Q2: If you did feel anxious, depressed or in any kind of mental distress, where would you go for help?

Select as many as are applicable
Q3: If you did feel anxious, depressed or in any kind of mental distress, would you Feel that you could approach your supervisor? Select only one answer
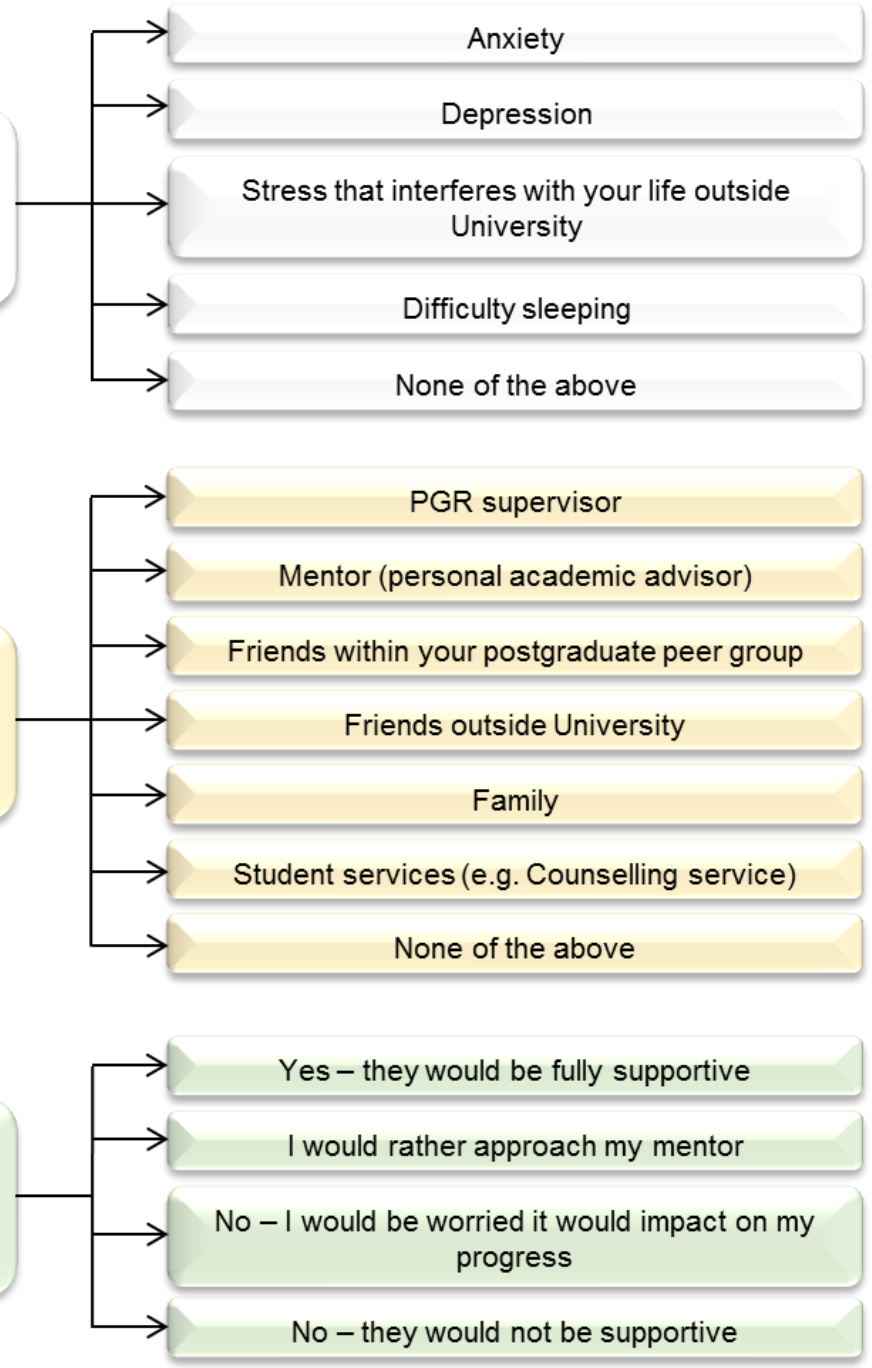

Q4: Are you aware of the well-being services that the university has for postgraduate students?

Select only one answer

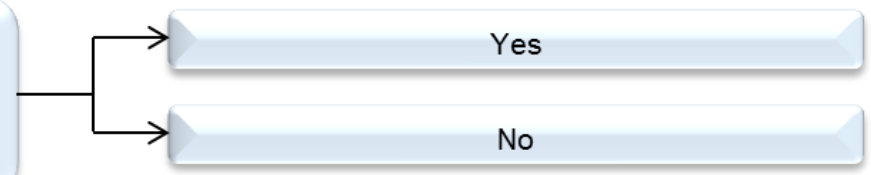

Q5: How do you think that the university could improve mental well-being of postgraduate students?

Free-form answer 


\section{Results}

\section{Response rate}

Of the 320 PGR students invited to take the survey, 54 responded (16.9\%). This could not be broken down further by faculty, gender or domestic/international status as this demographic data was not collected.

\section{Self-reported examples of poor mental health}

Only $9.3 \%$ of respondents reported no experiences of mental distress. Difficulty sleeping was the most prevalent problem $(75.9 \%)$ closely followed by stress that interferes with life outside the university $(72.2 \%)$ and anxiety (66.7\%). Depression was the lowest reported example but was still applicable to $42.6 \%$ of students (Figure $2 \mathrm{~A}$ ).

Figure 2. Features of poor mental health in PGR. (A) Survey respondents were asked to self-report whether they had experienced any features of poor mental wellbeing throughout their studies. (B) The number of students reporting from 0 to 4 experiences of poor mental well-being.

A

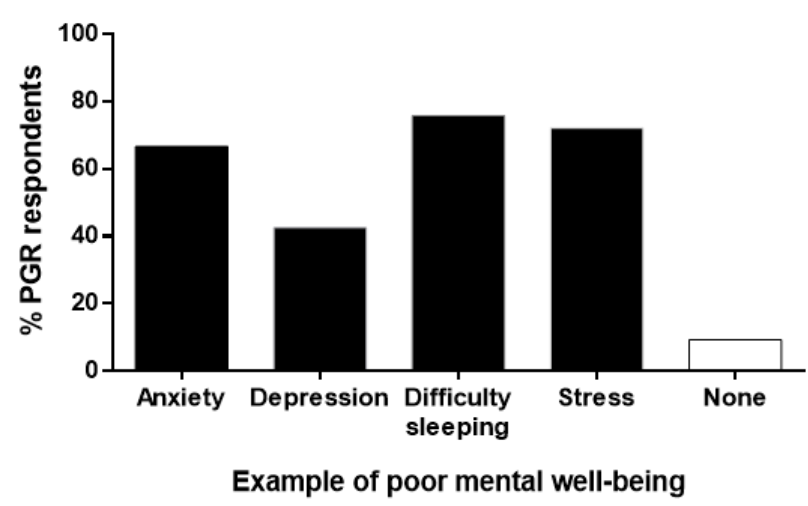

B

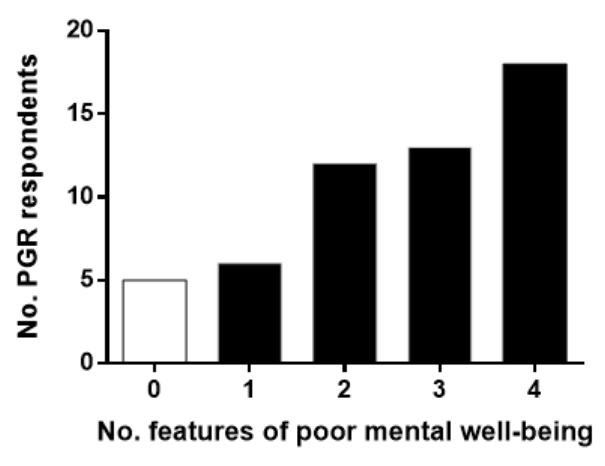

Students were able to select multiple options. Of the 49 students who reported problems with mental health, 6 selected only one option (with no single example standing out as prevalent), 12 selected two, 13 selected three and 18 had all four (Figure 2B), suggesting that students who did self-report tended towards more complex mental health issues. It is important to place this result in the context of response bias, as it may be likely that those who chose to respond already had experience of poor mental health, whereas those without may not have replied to our survey. 


\section{Sources of support in times of psychological stress}

Sources of support were segregated into those within the university and those outside. The most obvious source of support would be the supervisor; however, only $33.3 \%$ of students selected this. A similar proportion (31.5\%) would access Student Services, and only $14.8 \%$ would approach their mentor. The biggest source of internal support were friends within their postgraduate peer-group; however, this still only accounted for $37.0 \%$ of respondents. Students were much more likely to seek help outside the academic environment, with $51.9 \%$ indicating they would approach their family, and $61.1 \%$ their friends outside of university. However, of all respondents, 9.3\% would not approach any of the aforementioned sources of support if they were experiencing mental distress.

Whilst only a third of students selected the option that they would approach their supervisor, a significantly larger number (53.6\%) did feel that their supervisor would be fully supportive. Only 5.6\% would rather approach their mentor. Once again, however, this needs to be placed in context: our mentor scheme is new and in trial, with not all PGR students automatically receiving access to a mentor. This still left a substantial proportion of students (40.8\%) who would not speak to their supervisor, with the majority perceiving this would impact negatively on their progress (35.2\%). Only $5.6 \%$ of students stated that their supervisor would be unsupportive.

As previously stated, the university has multiple services including the counselling service and training courses on personal and professional development and resilience; however, only $51.9 \%$ were aware that the university had dedicated services for PGR. Clearly, we take from this that the learning developer needs to work even closer with faculty research leads and supervisors to improve awareness of the tailored support experience available.

\section{Suggestions for university provision of support}

Students were given free-form space to suggest areas in which the university could improve their mental health. The highlighted university-based causes of stress and possible solutions could be broadly split into 5 areas - university systems (e.g. PGR administration and facilities), supervisor training, well-being monitoring (e.g. regular contact to ascertain overall mental health needs), building networks, and finance. Of these, 
the first three received the highest volume of comment, and had substantial overlap

(Figure 3).

Figure 3. Areas of improvement identified by PGR. Venn diagram summarising freeform answers from the question 'How do you think that the university could improve the mental well-being of postgraduate students?'. answers coalesced into five interrelated areas: university systems, supervisor training, well-being monitoring, building networks, and finance. Comments were housed within each of these areas or applied across multiple areas as demonstrated. Text size is indicative of the frequency in which particular issues arose - more common suggestions are highlighted by larger text.

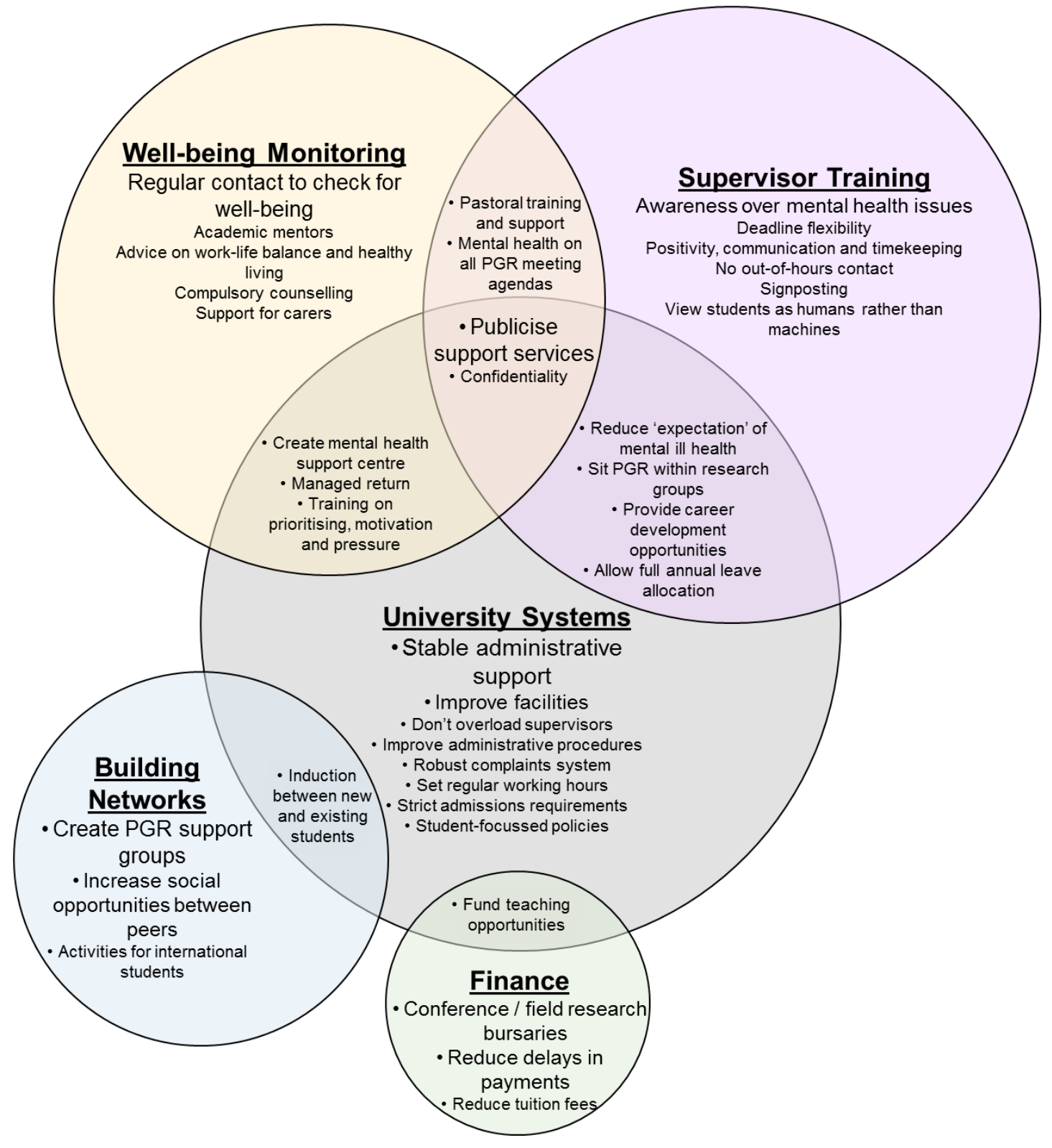


Four areas of improvement were suggested most frequently - providing stable administrative support (university systems), creating student support groups (building networks), for staff to regularly check for mental health (well-being monitoring) and to publicise the existing support services more widely (straddling well-being monitoring, supervisor training and university systems).

Some of the suggestions for improvement pertained to systems that are already in place at the university, for example, provision of academic mentors or training opportunities. The fact that these were given as areas of improvement validates the common suggestion to advertise these support services more widely and embed them further in the PGR experience. One respondent suggested:

Eliminate the culture of bad mental health where individuals who are currently not burnt out or suffering from mental illness are considered to be not working hard enough, and where the necessity of prescription drugs to alleviate anxiety and depression are currently seen as the norm.

Therefore, there is an awareness amongst the students that supervisors can be overloaded with teaching or supervisory responsibilities (university systems) and that academia often presents with a culture that rewards overworking (e.g. not taking annual leave, sending emails out of hours) where poor mental health is seen as the 'norm' (supervisor training).

\section{Discussion}

The impact of $\mathrm{HE}$ on mental health is increasingly well-recognised, with government policies recently announced to address the problems with stress and anxiety that reportedly afflict up to $17 \%$ of HEl students in the UK (Thorley, 2017). In keeping with Metcalfe et al. (2018), our study on mental health highlights the existence of poor mental health, and the difficulties inherent to academic institutions in terms of management, provision and finance. This poses a serious threat to the long-term mental health of graduates, both socially and psychologically, as it can impact their academic success (Eisenberg et al., 2009). 
The number of learners self-reporting experiences of poor mental health in our study is in keeping with Institute for Public Policy Research (Thorley, 2017), suggesting that HE culture needs to be assessed and revised to ameliorate these issues. Furthermore, our findings are in keeping with those of Metcalfe et al. (2018), who suggest the problem is escalating; our reported prevalence is higher than that reported in PRES (Slight, 2017).

\section{University systems and finance}

An issue emerging from our analysis is that of the processes and systems that underpin procedures for navigating doctoral learning. Transparent administrative processes can help students to feel in control and well prepared for key milestones and necessary bureaucratic hurdles. Most universities have administrative systems that are unique to PGR, given how different research degrees are from taught courses. Doctoral students are likely to seek guidance from their administrator(s) (Metcalfe et al., 2018), which was highlighted in our survey:

Our PGR admin had a crucial function: She could help students' mental health and ease students' anxiety because she was a person enough involved in . . . the PGR journey to understand sensitive supervision relationship dynamics, meaning she could provide pastoral support even when the supervisor relationship was problematic. ... At the same time [S] he was connected enough ... to provide timely assistance . . . for instance, the counselling service - even though very helpful in general - may not be able to.'

Conceptualised this way, admin is no longer 'admin' but part of the relationship-building with students who may want signposting to existing services and processes before raising things in research supervision. This has been configured as a positive and agentic aspect of students' confidence to look outside the doctoral relationship for relevant, timely support (McAlpine and McKinnon, 2013).

Related to this is the vital function of administrators in understanding and resolving financial aspects of daily living, project funding and fees. This finding is consistent with the role of financial (in)security found elsewhere (Ampaw and Jaeger, 2012), suggesting issues with money contribute to attrition and non-completion of doctoral programmes. Changes to administration exacerbate financial issues such as delayed subsistence payments or a lack of guidance for PGR in navigating the systems for managing fee 
payments, studentships and living allowances. The value of administration in securing a happy student experience is supported elsewhere (Cowling, 2017). Currently, the learning developer has regular and positive two-way interactions with PGR administration: directly supporting students and supervisors in accessing the wealth of information available through administration colleagues and actively taking referrals for support and development through administrators who work to ensure students access support when a need emerges. It is suggested that, given the importance attributed by respondents, these two-way relationships require further attention and resources.

\section{Publicity for existing systems}

Results indicate that most respondents would seek support outside of the university, with less than a third opting to access designated support services. This remains confusing as over half of respondents were aware of support services in the first place, leaving the question of why students would use outside services if it were not due to a lack of awareness of what is on offer. Indeed, due to the format of the survey, we cannot determine which support services students were aware of, nor why they opted not to access an internal service. It might be suggested that university services would benefit from greater awareness raising, including those offered by the PGR-dedicated learning developer. This is difficult, as it has been found that during induction programmes there was a perception that students were potentially receiving information when they least needed it (Metcalfe et al., 2018). Ergo, induction processes appear to be irrevocably flawed: presenting service information as part of an extended schedule of welcome and orientation events (the approach taken at our university) risks poor recall later in the learner's journey, as it may be shared at a time when personal value is not attributed to it.

Each university support service markets itself to students directly through a newsletter and online information that can be retrieved through the intranet upon searching. Faculty research student handbooks include service provision and contact details, and students undertake a training needs analysis as part of their initial supervisory engagement. As this is based on the Researcher Development Framework (Vitae, 2020b)(RDF is a UK framework that sets out professional skills and qualities development for all researchers, irrespective of career stage), it incorporates qualities, attributes and skills that can be developed that are more intrapersonal in nature, such as working on perseverance and 
resilience, including attending relevant training sessions that can help to develop these skills. Nevertheless, the national report highlights some of the issues with meaningful awareness raising of services that can support mental health. It is likely that our findings are consistent with this (Metcalfe et al., 2018, p.13). An action we have taken from this is to examine our taken-for-granted assumption that all PGR know enough about the internal support on offer to them at the different stages of their degree by research.

As a research team, we reflected that $\mathrm{HEl}$ experiences reproduce those found, anecdotally, outside of education, namely that signposting and preventative initiatives go unnoticed when an individual does not perceive a personal need, but only that individual would know when, in their learning journey, that point had been reached. Despite this, it is still something that our students feel that they would benefit from:

Give more information about the counselling service and the mental health advice that is part of disability services. It is important to know that other PhD students may be experiencing similar problems and these are not signs of weakness or poor academic ability.

There is currently no meaningfully reliable way to anticipate when any given student might benefit from mental health support, other than through interpersonal interaction with those important to the student's experience: supervisors, mentors, peers, and the learning developer. Empowering students to talk to those with whom they are in contact, in anticipation of mental health needs, may be the only way to effectively publicise pastoral and development services in a personally resonant way, especially when other students insist they want to know about everything on offer right from the beginning, rather than being 'drip-fed' information over time (Metcalfe et al., 2018).

The latest institutional figures also reveal the incongruity of our findings: on average $50 \%$ of new students attend all induction sessions. Could it be the case that our respondents suggesting a need for better awareness raising are those who do not engage in the preparatory, cohort-building classes? This may link to the point made above, that these are held within the first few weeks of learning (to build teams, help students to network and impart useful skills). Yet some may opt out due to it being too early for the value to be felt. As the learning developer runs our cross-institutional induction processes, further work is needed with research students and staff to identify how important support resources and 
services information can be made available on a needs basis. To date we have created an enhanced induction offer which supplies opportunities for networking and development over the first month of research (rather than a couple of concentrated days at the beginning) and worked with research leads and student evaluations to identify content for a PGR induction best-practice guide. Both initiatives are co-ordinated by the learning developer, who has regular contact with all parties involved, irrespective of research centre or faculty structures. This continues to be a work-in-progress.

\section{Building networks}

We recognise that less than $40 \%$ of respondents said they would turn to their peer networks for support. This is potentially of concern, as a similar proportion of respondents (just over 40\%) said they would not approach their supervisors, in which case, where are these students turning for support? The value of helping students build, maintain and utilise networks is a potential way forward in terms of working preventatively with students on their mental health (Hughes et al., 2018). This is, perhaps, under-utilised in our HEI currently. Peer-support schemes are becoming embedded at undergraduate level at the university, but a similar scheme for PGR is in its infancy. At present, only specific research groups have been selected for trial.

Linked to the importance of peer support networks is the very idea that a 'network' evokes, namely, that students are not actually alone, even if much time is spent on solitary scholarship; they can feel part of larger group of students all with a similar goal. As one of our respondents stated:

... as I am an international student, one of the reasons that worsen my anxiety, and depression is sometimes, especially at weekends. I do not find any one to go out with or talk to. If there would have been more activities for international students, I think that will help a lot.

When used effectively, there is evidence that feelings of isolation can be tackled through having a network to draw on and peer support is not only beneficial but essential (Solem et al., 2011). Even those who live away from campus can be connected to others electronically for 'moral' support, thinking and processing of frustrations, stressful experiences, failures and personal issues. Our survey, unlike Metcalfe et al. (2018) 
emphasises peer-to-peer student support, whereas the recent report also suggests that personal networks, unrelated to the daily pressures of the researcher, can provide relief, appropriate diversion and grounding, through continued investment in social or family life. This extends the idea that physical space to learn, work, and (importantly) connect during doctoral study is paramount, as students are embodied beings whose experiences are lived on and off campus, in relationship with others (Hopwood and Paulson, 2012). This may maintain perspective during anxiety- and stress-inducing periods at university.

As described above, inductions are essential but can be problematic in terms of timing and preferred content:

Have an induction between the newcomers and the previous PhD students, to help everyone integrate and feel wanted.

This student's perception indicates the beginning of the relational work that is needed, where individual learners are expected to develop and maintain fruitful relationships. The learning developer has worked with research leads to adapt our induction process following this, with existing research students meeting newcomers. He has also used student feedback to develop further induction and post-induction activities with the peerassisted learning team in professional services. This means students encounter a range of introductory sessions designed to build relationships and interpersonal skills, in addition to more traditional research skills, in the hope of offering a more rounded experience, with better integration and personal development (Hopwood and Paulson, 2012). We now need to monitor whether this has a positive impact.

\section{Well-being monitoring and supervisor training}

It was decided to name this area based on the language used by respondents: well-being. Well-being monitoring requires overarching support across the $\mathrm{HEI}$ and should not be left solely to the supervisor, who may well be untrained and unfamiliar in this area. Instead, a network led by the learning developer, comprising students, supervisors, and administrative support is necessary to fully support positive mental health. At our university, this has led to the development of a multi-voiced strategy to this effect. 
Successful relationships between supervisors and students are critical to the student experience. Currently, all supervisors undergo a one-day training course on university procedures and how to supervise doctoral students, with some emphasis on scenarios in which students are struggling. However, this training could be expanded specifically to address the growing awareness around mental health. Whilst supervisors are well versed in the need to monitor achievement over time, it can be argued that it is equally important to consider if PGR are happy, content and feeling valued, as this may indicate coping with the demands of doctoral study and that they are likely to be more productive than those who are not (Howells et al., 2017). Arguably, these students want their supervisors to broach these topics with them:

Talk more about it [mental health and well-being] especially by training supervisors to be approachable and watch out for signs and encourage students to seek help when they need it.

This reflects a resistance to the normalised discourses in doctoral learning, in which intrapersonal suffering and being left to deal with issues alone are prevalent (John and Denicolo, 2013).

One source of conflict is a mismatch of expectation, both from the point of view of the student and of the supervisor. In such cases, expectation management on both sides very early on in the process is crucial in order to facilitate a harmonious and productive relationship (Bui, 2015); however, this is known to often be disjunctive (Malfroy, 2005). Anecdotally, students can have unrealistic expectations of the amount of time they will spend with their supervisor, and the role that they will play in the wider research group. As an example, students often imagine that their role is strictly to answer their research 'question'. However, in reality, a PhD is a training degree bestowing the student with essential transferable skills for independence and future employment. This includes time management, prioritisation and peer training in addition to their research project. Thus, emphasising this level of independence in initial supervisory meetings, the induction process and peer-support networks would be beneficial, as students' expectations may need to be managed in terms of both supervisor contact and the nature of degrees by research. This notion of expectation management also occurs when developing research supervisors. Supervisors can have unrealistic expectations regarding students' abilities and workloads, which often lead to conflict. Students have reported this as: 
For the institution to remember students are human and not thesis machines.

This has been explored in supervision research as an issue of 'contradiction and congruence', whereby a supervisor may wish to facilitate autonomy, academic exploration, and personalisation of learning (Delderfield and McHattie, 2018). However, supervisors may be subject to pressures to adhere to completion rates and other research metrics that place the obtaining of outputs ahead of the human experience of facilitated learning (Deuchar, 2008). As well as the increasing prevalence of mental ill-health in PGR, there is a growing recognition of psychological issues throughout academia including, crucially, supervisors. Recent large-scale studies in The Guardian and Times Higher Education have highlighted issues with anxiety (82\%), depression (74\%; Thomas, 2014) and sleeplessness (up to 55\%; Grove, 2018) in UK academics. Stressors include juggling everincreasing workloads and roles, including administrative duties that are not considered traditionally 'academic' jobs and with which research-focused academics are not familiar or confident.

Although this culture of mental ill-health in academics has long been recognised (Kinman, 2008; Pells, 2018), it has received limited attention, with mental health interventions in HEls usually focussing solely on student experiences. However, it is interesting to note that stressors for academics include high self-expectation, lack of support, poor remuneration and poor work-life balance (Kinman, 2008; Sang et al., 2015) - factors which mirror concerns for students. It is possible that, as academics are routinely experiencing these stressors themselves, they are inadvertently perpetuating the cycle of poor mental health and work-life balance among the next generation. It may be that until mental health in supervisors is addressed, there will be no improvement in the mental health of students. This is recognised by respondents:

I do think the culture of academia currently is pressured and we see University staff working with large workloads. ... There seems to be a culture of expectation to work unsocial hours, for example emails being sent out late in the evenings and weekends. This can make you feel that . . . you don't want to work where this is expected as it is an unhealthy work-life balance.

Being realistic and transparent about expectations from all sides (student, supervisor and institution) is subject to current research. For example, routine over-work and a non- 
individualised, one-size-fits-all approach to doctoral learning are beginning to be resisted (Parker-Jenkins, 2018). At our university, the learning developer is in an optimal position to use his situatedness in the 'third space' (Whitchurch, 2013) and the RDF to frame advanced training for all researchers from PGR to experienced supervisors in order to improve the training and one-to-one support offer pertaining to enhanced skills for supervision. This cross-over work between LD, human resources, and staff research services is trialling this new training to address supervisors' ongoing development. In addition, teaching was added to induction fostered around the objective of successful working with supervisors for new PGR who wanted to learn more about the supervisory relationship. This means systemic challenge and change to staff skills and research culture can be collaboratively achieved (Parkes, 2018), whilst serving the transient population of research students year-on-year.

\section{Limitations, future work and conclusions}

There are several limitations that need to be borne in mind when interpreting our findings. The design of the study was necessarily pragmatic, as it arose from an opportunity to ask questions of our learners at a time when academics and learning developers were meeting to discuss this group of students. The sample size is small, with no additional contextualising demographic data that might allow issues around features such as ethnicity or gender to be drawn out. Response bias also needs to be considered. As stated above, it is likely that respondents replied to our call as they had a personal interest or experience in mental health. At its most fundamental level, the small sample and unvalidated survey mean we have no way to determine the relative integrity of students' responses.

We also recognise that the questions asked include demand characteristics, whereby our respondents could take their cue from the purpose of the survey and the framing of the question, choosing to respond in a certain manner emphasising problematic issues rather than positive mental health. In these respects, our survey is inferior to the validated instrument developed for use with doctoral researchers mentioned previously (Juniper et al., 2012), yet has still provided us with a useful indication of the mental health concerns of our students, of which we would otherwise have remained ignorant. 
This study emulates more modest studies (e.g. Brown and Watson, 2010) by focussing on PGR within a single HEl. It has provided us with a snapshot of mental health among our learners and has highlighted several areas which can be improved in order to enhance their experience, these being: university systems, supervisor training, well-being monitoring, building networks, and finance (Figure 3). Should the survey run in the future, to give a more exhaustive account of mental health and the issues facing PGR, we would make significant amendments. These relate to the methodological robustness of its design. The survey does not currently allow students to identify whether they had preexisting poor mental health or whether this was exacerbated by (or originated during) their postgraduate studies. We recognise that the survey response rate is quite low, although the data collated is from 54 respondents. In the future, we would leave the survey open for one month and send weekly reminders to PGR requesting they fill in the survey to increase our sample size. Further research would benefit from focussing across multiple sites to ascertain the universality of declining mental health in doctoral learners. There is also potential for a reflective study on the collaborative relationship between academic research supervisors and learning developers, something that deserves further attention.

Notwithstanding these shortcomings, our modest engagement does have merits. Knowledge is sparse in this area, and our site-specific data are the first of their kind. Our research supports recent UK findings about PGR mental health discussed above (Metcalfe et al., 2018) and, whilst we do not itemise specific recommendations at this exploratory stage, the insights here form the basis of potential future work at our university. Examples of these include: working to deliver more to address the well-being aspects of the RDF; continued work on PGR induction; developing our institutional PGR strategy (led by our learning developer), the PGR experience of which involves the five areas gleaned from our work; and, lastly, continuing our work to improve the training of mentors and research supervisors. This endeavour is shared between the research leads and the learning developer, who, of all colleagues, continues to be ideally placed and professionally skilled to work across institutional boundaries and structures to deliver changes for individuals and the organisation. This is our first foray into capturing the meanings and relationships between LD, PGR and mental health. Our institutional changes are fresh and, as yet, unreported. Thus, evaluations of this work are likely to be available in due course. 


\section{References}

Ampaw, F. D. and Jaeger, A. J. (2012) 'Completing the three stages of doctoral education: An event history analysis', Research in Higher Education, 53(6), pp.640-660.

Braun, V. and Clarke, V. (2006) 'Using thematic analysis in psychology', Qualitative Research in Psychology, 3(2), pp.77-101.

Brown, L. and Watson, P. (2010) 'Understanding the experiences of female doctoral students', Journal of Further and Higher Education, 34(3), pp.385-404.

Bui, H. T. M. (2015) 'Student-supervisor expectations in the doctoral supervision process for business and management students', Business and Management Education in Higher Education, 1(1), pp.12-27.

Cairns, J., Hervey, T. and Johnson, O. (2018) 'Neither "bolt-on" nor "built-in": benefits and challenges of developing an integrated skills curriculum through a partnership model', Journal of Learning Development in Higher Education. (13)1, pp.1-22. Available at: https://journal.aldinhe.ac.uk/index.php/ildhe/article/view/435 (Accessed: 15 August 2020).

Cowling, M. (2017) Happiness in UK postgraduate research: an analysis of results from the Postgraduate Research Experience Survey. Available at:

https://www.heacademy.ac.uk/knowledge-hub/happiness-uk-post-graduateresearch-uk-heis (Accessed: 3 September 2018).

Delderfield, R. and McHattie, H. (2018) 'Person-centred dynamics in maths skills development: examining a case of good practice', Journal of Learning Development in Higher Education, 13(1), pp.1-10. Available at:

https://journal.aldinhe.ac.uk/index.php/jldhe/article/view/447 (Accessed: 14 January 2020).

Deuchar, R. (2008) 'Facilitator, director or critical friend?': Contradiction and congruence in doctoral supervision styles, Teaching in Higher Education, 13(4), pp.489-500. 
Dhillon, S. (2018) 'Whose wellbeing is it anyway?', Journal of Learning Development in Higher Education, 13(2). Available at:

https://journal.aldinhe.ac.uk/index.php/ildhe/article/view/460 (Accessed: 30 July 2020).

Editorial (2018) 'Time to talk about why so many postgrads have poor mental health', Nature, 556(5). Available at: https://www.nature.com/articles/d41586-018-04023-5 (Accessed: 15 October 2018).

Eisenberg, D., Golberstein, E. and Hunt, J.B. (2009) 'Mental health and academic success in college', The B. E. Journal of Economic Analysis and Policy, 9(1), pp.1-37.

Evans, T. M., Bira, L., Gastelum, J. B., Weiss, L. T. and Vanderford, N. L. (2018) 'Evidence for a mental health crisis in graduate education', Nature Biotechnology, 36(3), pp.282-284.

Gorczynski, P., Sims-Schouten, W., Hill, D. and Wilson, J. C. (2017) 'Examining mental health literacy, help seeking behaviours and mental health outcomes in UK university students', The Journal of Mental Health Training, Education and Practice, 12(2), pp.111-120.

Grove, J. (2018) 'Half of UK academics suffer stress-linked mental health problems', Times Higher Education 6 July 2018. Available at: https://www.timeshighereducation.com/news/half-uk-academics-suffer-stresslinked-mental-health-problems (Accessed: 15 October 2018).

Guthrie, S., Lichten, C. A., Van Belle, J., Ball, S., Knack, A. and Hofman, J. (2017) 'Understanding mental health in the research environment: A rapid evidence assessment', Rand Health Quarterly, 7(3). Available at: https://www.rand.org/pubs/research reports/RR2022.html (Accessed: 15 October 2018). 
Hopwood, N. and Paulson, J. (2012) 'Bodies in narratives of doctoral students' learning and experience', Studies in Higher Education, 37(6), pp.667-681.

Howells, K., Stafford, K., Guijt, R. and Breadmore, M. (2017) 'The role of gratitude in enhancing the relationship between doctoral research students and their supervisors', Teaching in Higher Education, 22(6), pp.621-638.

Hughes, G., Panjawni, M., Tulcidas, P. and Byrom, N. (2018) 'Student mental health: The role and experiences of academics', Student Minds. Available at: https://www.studentminds.org.uk/theroleofanacademic.html (Accessed: 29 September 2018).

John, T. and Denicolo, P. (2013) 'Doctoral education: a review of the literature monitoring the doctoral student experience in selected OECD countries (mainly UK)', Springer Science Reviews, 1, pp.41-49. Available at: https://doi.org/10.1007/s40362-0130011-x (Accessed: 29 September 2018).

Johnson, I. (2018) 'Driving learning development professionalism forward from within', Journal of Learning Development in Higher Education, Special Edition: ALDinHE Conference 2018, pp.1-29. Available at:

https://journal.aldinhe.ac.uk/index.php/jldhe/article/view/470 (Accessed: 19 August 2020).

Juniper, B., Walsh, E., Richardson, A. and Morley, B. (2012) 'A new approach to evaluating the well-being of PhD research students', Assessment and Evaluation in Higher Education, 37(5), pp.563-576.

Kinman, G. (2008) 'Work stressors, health and sense of coherence in UK academic employees', Educational Psychology, 28(7), pp.823-835.

Levecque, K., Anseel, F., De Beuckelaer, A., Van der Heyden, J. and Gisle, L. (2017) 'Work organization and mental health problems in PhD students', Research Policy, 46(4), pp.868-879. 
Maguire, M. and Delahunt, B. (2017) 'Doing a thematic analysis: A practical, step-by-step guide for learning and teaching scholars', AISHE-J: The All Ireland Journal of Teaching and Learning in Higher Education, 9(3), pp.1-14.

Malfroy, J. (2005) 'Doctoral supervision, workplace research and changing pedagogic practices', Higher Education Research and Development, 24(2), pp.165-178.

Mascaskill, A. (2012) 'University student mental health in crisis: A United Kingdom perspective', British Journal of Guidance Counselling, 41(4), pp.426-441.

Mental Health Foundation (2018) Your mental health. Available at: https://www.mentalhealth.org.uk/your-mental-health (Accessed: 6 February 2020).

Metcalfe, J., Wilson, S. and Levecque, K. (2018) Exploring wellbeing and mental health and associated support services for postgraduate researchers. Cambridge: Careers Research and Advisory Centre. Available at: https://www.vitae.ac.uk/doingresearch/wellbeing-and-mental-health/HEFCE-Report Exploring-PGR-Mentalhealth-support/view (Accessed: 3 May 2018).

McAlpine, L. and McKinnon, M. (2013) 'Supervision - the most variable of variables: student perspectives', Studies in Continuing Education, 35(3), pp.265-280.

Mortier, P., Auerbach, R.P., Alonso, J., Axinn, W.G., Cuijpers, P., Ebert, D.D., Green, J.G., Hwang, I., Kessler, R.C., Liu, H., Nock, M.K., Pinder-Amaker, S., Sampson, N.A., Zaslavsky, A.M., Abdulmalik, J., Aguilar-Gaxiola, S., Al-Hamzawi, A., Benjet, C., Demyttenaere, K., Florescu, S., De Girolamo, G., Gureje, O., Haro, J.M., Hu, C., Huang, Y., De Jonge, P., Karam, E.G., Kiejna, A., Kovess-Masfety, V., Lee, S., Mcgrath, J.J., O'neill, S., Nakov, V., Pennell, B.E., Piazza, M., Posada-Villa, J., Rapsey, C., Viana, M.C., Xavier, M. and Bruffaerts, R. (2018) 'Suicidal thoughts and behaviors among college students and same-aged peers: results from the World Health Organization World Mental Health Surveys', Social Psychiatry and Psychiatric Epidemiology, 53(3), pp.279-288. 
Neves, J. and Hillman, N. (2017) Student academic experience survey, Higher Education Policy Institute. Available at: https://www.hepi.ac.uk/2017/06/07/2017-studentacademic-experience-survey/ (Accessed: 15 January 2020).

Pain, E. (2018) 'Graduate students need more mental health support, new study highlights', Science (blog). Available at:

https://www.sciencemag.org/careers/2018/03/graduate-students-need-more-mentalhealth-support-new-study-highlights (Accessed: 15 January 2020).

Parkes, S. (2018) 'A learner developer perspective: critiquing dominant practices and cultures within university spaces', Journal of Learning Development in Higher Education, Special Edition: ALDinHE Conference 2018, pp.1-13. Available at: https://journal.aldinhe.ac.uk/index.php/jldhe/article/view/464 (Accessed: 18 August 2020).

Parker-Jenkins, M. (2018) 'Mind the gap: developing the roles, expectations and boundaries in the doctoral supervisor-supervisee relationship', Studies in Higher Education, 43(1), pp.57-71.

Pells, R. (2018) 'Lecturer's suicide a 'wake-up call' on overworking in academia', Times Higher Education 14 ${ }^{\text {th }}$ June (Accessed: $28^{\text {th }}$ July 2020).

Pole, C. J., Sprokkereef, A., Burgess, R. G. and Lakin, E. (1997) 'Supervision of doctoral students in the natural sciences: Expectations and experiences', Assessment and Evaluation in Higher Education, 22(1), pp.49-63.

Sang, K., Powell, A., Finkel, R. and Richards, J. (2015) 'Being an academic is not a 9-5 job: long working hours and the ideal worker in UK academia', Labour and Industry: A Journal of the Social and Economic Relations of Work, 25(3), pp.235-249.

Slight, C. (2017) Postgraduate Research Experience Survey 2017: experiences and personal outlook of postgraduate researchers. Higher Education Academy. Available at: https://www.heacademy.ac.uk/knowledge-hub/postgraduate-researchexperience-survey-report-2017 (Accessed: 15 October 2019). 
Solem, M., Hopwood, N. and Schlemper, B. (2011) 'Experiencing graduate school: A comparative analysis of students in geography programs', The Professional Geographer, 63(1), pp.1-17.

Stapleford, K. (2019) 'The LDHEN hive mind: Learning Development in UK higher education as a professional culture'. Journal of Learning Development in Higher Education, 16(1), pp.1-23. Available at: https://journal.aldinhe.ac.uk/index.php/ildhe/article/view/510 (Accessed: 1 January 2020).

Thomas, K. (2014) 'We don't want anyone to know, say depressed academics', The Guardian HE, 8 May. Available at: https://www.theguardian.com/higher-educationnetwork/blog/2014/may/08/academics-mental-health-suffering-silence-guardiansurvey (Accessed: 13 October 2019).

Thorley, C. (2017) Not by degrees: improving student mental health in the UK's universities. Institute for Public Policy Research (September). Available at: www.ippr.org/publications/not-by-degrees (Accessed: 28 July 2020).

Universities UK (2017) Step Change: mentally healthy universities. Available at: https://www.universitiesuk.ac.uk/stepchange (Accessed: 15 October 2019).

Vitae (2020a) Evolution of the Concordat. Available at: https://www.vitae.ac.uk/policy/concordat/background (Accessed: 10 September 2020).

Vitae (2020b) The Vitae Researcher Development Framework. Available at: https://www.vitae.ac.uk/researchers-professional-development/about-the-vitaeresearcher-development-framework/developing-the-vitae-researcher-developmentframework (Accessed: 10 September 2020).

Whitchurch, C. (2013) Reconstructing identities in higher education: the rise of 'third space' professionals. London: Routledge. 
World Health Organization. (2014) Social determinants of mental health. Available at: http://www.who.int/mental health/publications/gulbenkian paper social determinan ts of mental health/en/ (Accessed: 15 October 2019).

\section{Author details}

Russell Delderfield is PGR Framework Lead at the University of Bradford.

Kirsten Riches-Suman is a lecturer in Biomedical Science at the University of Bradford.

Mathias Ndoma-Egba is a postgraduate researcher in the Faculty of Management, Law and Social Sciences at the University of Bradford.

James Boyne is a reader in Molecular Biology at the University of Huddersfield. 